
\title{
25 Research Soure \\ Time-Series Analysis of Coxsackievirus B Serotype Surveillance Data in Japan
}

\section{Ayako Sumi ( $\nabla$ sumi@sapmed.ac.jp )}

Department of Hygiene, Sapporo Medical University School of Medicine https://orcid.org/0000-00025347-650X

\section{Keiji Mise}

Department of Admission and High school Liaison, Center for Medical Education, Sapporo Medical Univsesity

\section{Research note}

Keywords: Type 1 diabetes, Coxsackievirus B, time-series analysis, periodicity, surveillance, Japan

Posted Date: March 9th, 2021

DOl: https://doi.org/10.21203/rs.3.rs-286112/v1

License: @ (i) This work is licensed under a Creative Commons Attribution 4.0 International License. Read Full License 


\section{Abstract}

Objective

Studies have identified serotypes of Coxsackievirus B (CVB) enterovirus as a cause of type 1 diabetes. Studies have also identified cyclical variations in type 1 diabetes incidence-peak incidences occurring in 4- to 6-years periods in two regions in England, a 5-year period in Western Australia, and 5.33-year period in Poland. To date, no studies have investigated whether CVB infection rates demonstrate similar cyclical variation characteristics. The purpose of this study was to characterize periodicity in CVB surveillance data.

Results

Maximum entropy spectral analysis was performed on monthly CVB surveillance data. In addition to demonstrating a 1-year cycle for all serotypes, spectral peaks demonstrated dominant cycles-6.9-, 3.8-, 4.3-, 9.5-, and 7.8- year periods for CVB1, CVB2, CVB3, CVB4, and CVB5, respectively. Pearson correlation was used to compare the least-squares fit curves based on periods estimated from the analysis with the original data. The results for all five serotypes-CVB1, CVB2, CVB3, CVB4, and CVB5-demonstrated good correlation $-\rho=0.96, \rho=0.60, \rho=0.90, \rho=0.88$, and $\rho=0.67$, respectively. This method could be a useful tool for the efficient investigation of CVB as a pathogen of type 1 diabetes.

\section{Introduction}

Coxsackie B (CVB) enterovirus serotypes have recently attracted attention as a cause of type 1 diabetes, which has a high incidence high among children in European countries [1,2]. The estimated increase in annual incidence of type 1 diabetes in Europe was 3.9\% (95\% Cl 3.6\%, 4.2\%) from 1989 to 2003; worldwide, the estimated annual increase was $2.8 \%(95 \% \mathrm{Cl} 2.4 \%, 3.2 \%)$ from 1990 to 1999 [3].

Examining the periodic structure of CVB serotype surveillance data is essential for predicting the epidemic of type 1 diabetes. Some researchers have reported cyclical variations in yearly incidence rates of type 1 diabetes-4-year intervals in the Yorkshire region in England from 1978 to1990 [4], a 6-year cyclical pattern in a neighboring area of northeast England from 1990 to 2007 [5], a sinusoidal cycle with peaks every 5 years in Western Australia from 1985 to 2010 [6], and a 5.33-year periodicity in Poland during the period 1989-2012 [7]. More recently, to help clarify recent trends in European incidence rates, European Diabetes registry data were analyzed from over 84,000 children in 26 European centers representing 22 countries from 1989-2013, with separate estimates of incidence rate increases derived in five subperiods [3].

To date, no studies have examined whether surveillance data for CVB serotypes show similar cycles as those in type 1 diabetes incidence data, likely because studies investigating publicly available CVB serotype surveillance data for Europe are lacking. On the other hand, in Japan, CVB serotype surveillance data has been collected for 20 years [8]. The purpose of this study was to investigate the periodic 
structure of Japanese CVB serotype surveillance data of using time-series analysis based on the maximum entropy method (MEM) in the frequency domain and the least squares method (LSM) in the time domain $[9,10]$.

\section{CVB Serotype Surveillance}

Monthly surveillance data of CVB serotypes (CVB1, CVB2, CVB3, CVB4, and CVB5) from January 2000 to December 2018 (228 data points) were analyzed. The number of specimens that test positive for pathogens and viruses, including CVB serotypes, are regularly reported to the National Institute of Infectious Disease Surveillance Center (Tokyo, Japan). These data are published in the monthly periodical Infectious Agents Surveillance Report [11].

Monthly surveillance data of CVB serotype from January 2000 to December 2018 are shown in Figure 1. Therein, all incidence data show a yearly cycle with large epidemics every few years, for example, CVB1 (Figure 1a) in 2004 and 2011 and CVB2 (Figure 1c) in 2005 and 2009.

\section{Periodicity of the Surveillance Data}

Power spectral densities (PSDs) obtained with the MEM spectral analysis (Additional file 1) for the data in Figure 1 are shown in Figure 2. In each plot- CVB1 (Figure 2a), CVB2 (Figure 2b), CVB3 (Figure 2c), CVB4 (Figure 2d), and CVB5 (Figure 2e)-prominent spectral peaks were observed at $f=1.0$ [units (1/year)], corresponding to the 1 -year cycle, that is, the seasonal cycle. In the low-frequency range, $f<1.0$, reflecting oscillations longer than the 1-year cycle, several prominent spectral peaks were observed. In each power spectral density plot, the dominant spectral peak was observed during an approximately 3 - to 5 -year period. For each serotype, five dominant spectral frequency mode peaks with corresponding periods and powers are listed in Table 1.

With the five periodic modes that were clearly observed in each PSD (Table 1), the least squares fitting (LSF) curve (Additional file 2) for each serotype was calculated. Each LSF curve thus obtained is presented in Figure 1

Each LSF curve reproduced the original data well (Figure 1), which confirmed the periods from MEM spectral analysis (Figure 2, Table 1) were accurate. Pearson correlations between the original data and the LSF curves $-\rho=0.96, \rho=0.60, \rho=0.90, \rho=0.88$, and $\rho=0.67$ for CVB1, CVB2, CVB3, CVB4 and CVB5, respectively-further demonstrated a good fit.

\section{Discussion And Conclusions}

An important finding of this study was the 3- to 5-year period in enterovirus surveillance data in Japan (Figure 2 and Table 1). This period is similar to that observed in time-series data on the number of patients with type 1 diabetes in Europe [2]. Therefore, if periodicities in CVB infection rates similar to those identified in these surveillance data in Japan can be found in European data, it would support the association between CVB serotypes and type 1 diabetes. Countries with large numbers of patients with 
type 1 diabetes, such as Finland, have published surveillance data for enteroviruses but not for subtypespecific enterovirus. To resolve the high incidence of type 1 diabetes in Europe, access to subtype-specific enterovirus surveillance data is essential. We anticipate that this method of time-series analysis will be a useful tool for elucidating periodicity in subtype-specific enterovirus surveillance data.

\section{Limitation}

A limitation of this study was that a direct comparison between CVB infection rate and type 1 diabetes periodicities could not be performed since we did not have access to CVB epidemiological time-series data for European countries. Investigating the correlation of CVB infection rates with type 1 diabetes, for example in countries such as Finland, would allow efficient estimation CVB as pathogen of type 1 diabetes, to help reduce and prevent type 1 diabetes.

\section{Abbreviations}

LSF, least squares fitting; MEM, maximum entropy method; PSD, power spectral density.

\section{Declarations}

\section{Ethics approval and consent to participate}

Not applicable

\section{Consent for publication}

Not applicable.

\section{Availability of data and material}

The dataset of surveillance data analyzed during the current study are available from ref. [9].

\section{Competing interests}

We declare that I have no competing interests.

\section{Funding}

This research was funded by the Japan Society for the Promotion of Science KAKENHI grant number $19 \mathrm{~K} 10666$.

\section{Author's contributions}

Both authors conceived the study and managed the data. KM conceived the study and drafted the manuscript. AS analyzed the data and wrote the final version of this paper, read the final manuscript and it. 


\section{Acknowledgements}

We thank Coren Walters-Stewart, PhD from Edanz Group (https://en-author-services.edanzgroup.com/ac) for editing a draft of this manuscript and for helping to draft the abstract.

\section{References}

1. Rewers M, Ludvigsson J. Environmental risk factors for type 1 diabetes.Lancet 2016; 387: 23402348.

2. Patterson CC, Karuranga S, Salpea P, Saeedi P, Dahlquist G, Soltesz G, Ogle GD. Worldwide estimates of incidence, prevalence and mortality of type 1 diabetes in children and adolescents: Results from the International Diabetes Federation Diabetes Atlas, 9th edition.Diabetes Res Clin Pract 2019; 157: 107482-107490.

3. Patterson CC, Harjutsalo V, Rosenbauer J, Neu A, Cinek O, Skrivarhaug T, et al. Trends and cyclical variation in the incidence of childhood type 1 diabetes in 26 European centres in the 25 year period 1989-2013: a multicentre prospective registration study. Diabetologia 2019; 62: 408-417.

4. Staines A, Bodansky HJ, Lilley HE, Stephenson C, McNally RJ, Cartwright RA. The epidemiology of diabetes mellitus in the United Kingdom: the Yorkshire Regional Childhood Diabetes Register.Diabetologia 1993; 36: 1282-1287.

5. McNally RJ, Court S, James PW, Pollock R, Blakey K, Begon M. Cyclical variation in type 1 childhood diabetes. Epidemiology 2010; 21: 914-915.

6. Haynes A, Bulsara MK, Bower C, Jones TW, Davis EA. Cyclical variation in the incidence of childhood type 1 diabetes in Western Australia (1985-2010).Diabetes Care 2012; 35: 2300-2302.

7. Chobot A, Polanska J, Brandt A, Deja G, Glowinska-Olszewska B, Pilecki O, et al.Updated 24-year trend of type 1 diabetes incidence in children in Poland reveals a sinusoidal pattern and sustained increase. Diabet Med 2017; 34: 1252-1258.

8. Pons-Salort M, GrasslySerotype-specific immunity explains the incidence of diseases caused by human enteroviruses. Science 2018; 361: 800-803.

9. National Institute of Infectious Diseases. Infectious Agents Surveillance Report (https://www.niid.go.jp/niid/en/iasr.html). Accessed 3, December 2020.

10. Sumi A, Kobayashi N. Time-series analysis of geographically specific monthly number of newly registered cases of active tuberculosis in Japan. PLOS ONE 2019; 14: e0213856.

11. Sumi A, Toyoda S, Kanou K, Fujimoto T, Mise K, Kohei Y, et al. Association between meteorological factors and reported cases ofhand, foot, and mouth disease from 2000 to 2015 in Japan. Epidemiol Infect 2017; 145: 2896-2911.

12. Armitage P, Berry G, Matthews JNS. Statistical Method in Medical Research, 4th edn. Oxford: Blackwell Science, 2012. 
13. Ohtomo N, Terachi S, Tanaka Y, Tokiwano K, Kaneko N. New method of time series analysis and its application to Wolf's sunspot number data.Jpn J Appl Phys 1994; 33: 2321-2831.

14. Finnish Institute for Health and Welfare. Statistical Databases (https://thl.fi/en/web/thlfi-en). Accessed 3, December 2020.

\section{Table}

Table 1. Characteristics of the five dominant spectral peaks shown in Figure 2. 


\begin{tabular}{|c|c|c|c|}
\hline & Frequency (1/year) & Period (year) & Power \\
\hline \multirow[t]{5}{*}{ CoxB1 } & 0.15 & 6.88 & 18.52 \\
\hline & 0.29 & 3.46 & 15.07 \\
\hline & 0.85 & 1.18 & 1.18 \\
\hline & 0.99 & 1.01 & 16.1 \\
\hline & 1.15 & 0.87 & 6.61 \\
\hline \multirow[t]{5}{*}{ CoxB2 } & 0.26 & 3.80 & 12.97 \\
\hline & 0.47 & 2.14 & 5.85 \\
\hline & 0.53 & 1.90 & 4.17 \\
\hline & 0.74 & 1.35 & 4.42 \\
\hline & 1.00 & 1.00 & 14.32 \\
\hline \multirow[t]{5}{*}{ CoxB3 } & 0.23 & 4.31 & 25.63 \\
\hline & 0.46 & 2.19 & 9.2 \\
\hline & 0.75 & 1.33 & 7.74 \\
\hline & 1.01 & 1.00 & 32.28 \\
\hline & 1.25 & 0.80 & 10.31 \\
\hline \multirow[t]{5}{*}{ CoxB4 } & 0.11 & 9.48 & 6.93 \\
\hline & 0.26 & 3.92 & 2.69 \\
\hline & 0.89 & 1.12 & 3.73 \\
\hline & 1.00 & 1.01 & 25.26 \\
\hline & 1.98 & 0.50 & 5.35 \\
\hline \multirow[t]{5}{*}{ CoxB5 } & 0.13 & 7.83 & 32.09 \\
\hline & 0.24 & 4.13 & 46.68 \\
\hline & 0.68 & 1.48 & 19.78 \\
\hline & 1.01 & 0.99 & 53.25 \\
\hline & 1.25 & 0.80 & 18.79 \\
\hline
\end{tabular}

Figures 

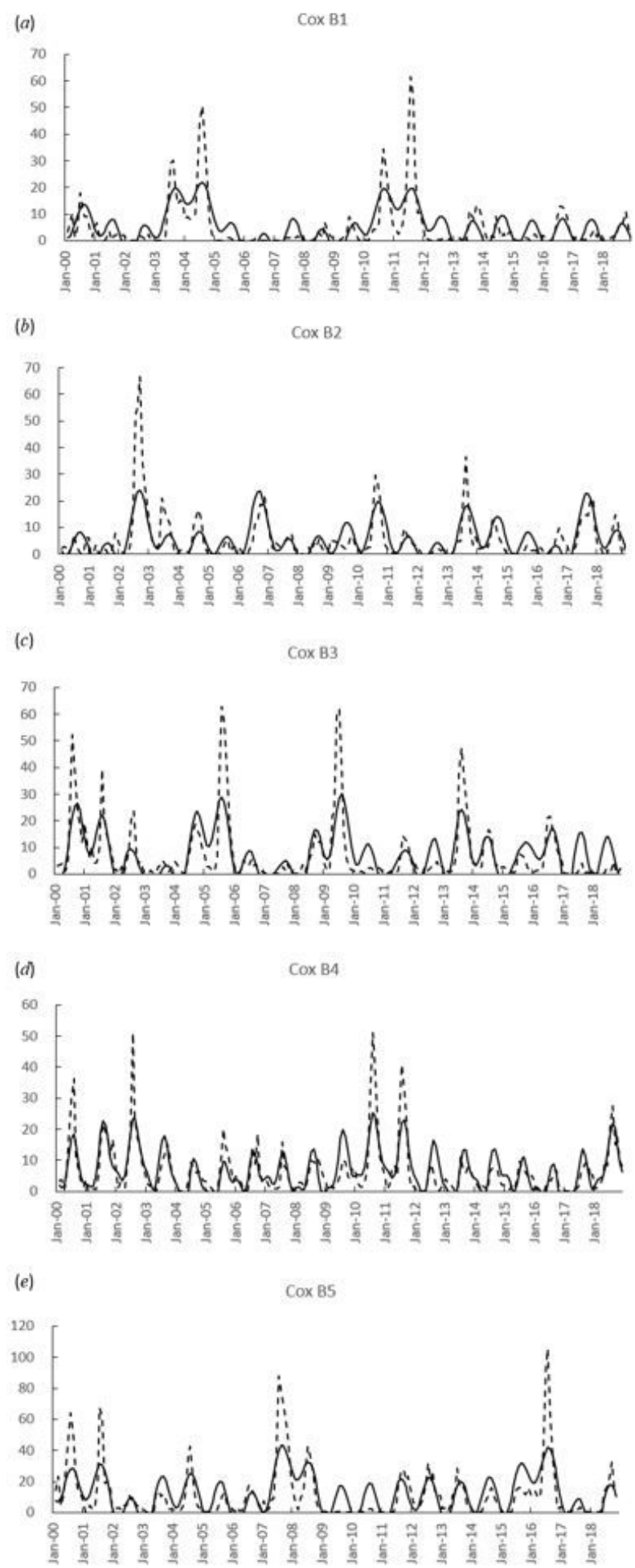

\section{Figure 1}

Comparison of the least-squares fitting curves calculated for long-term trends (solid line) in with original data (dotted line) for (a) CVB1, (b) CVB2, (c) CVB3, (d) CVB4, and (e) CVB5. 


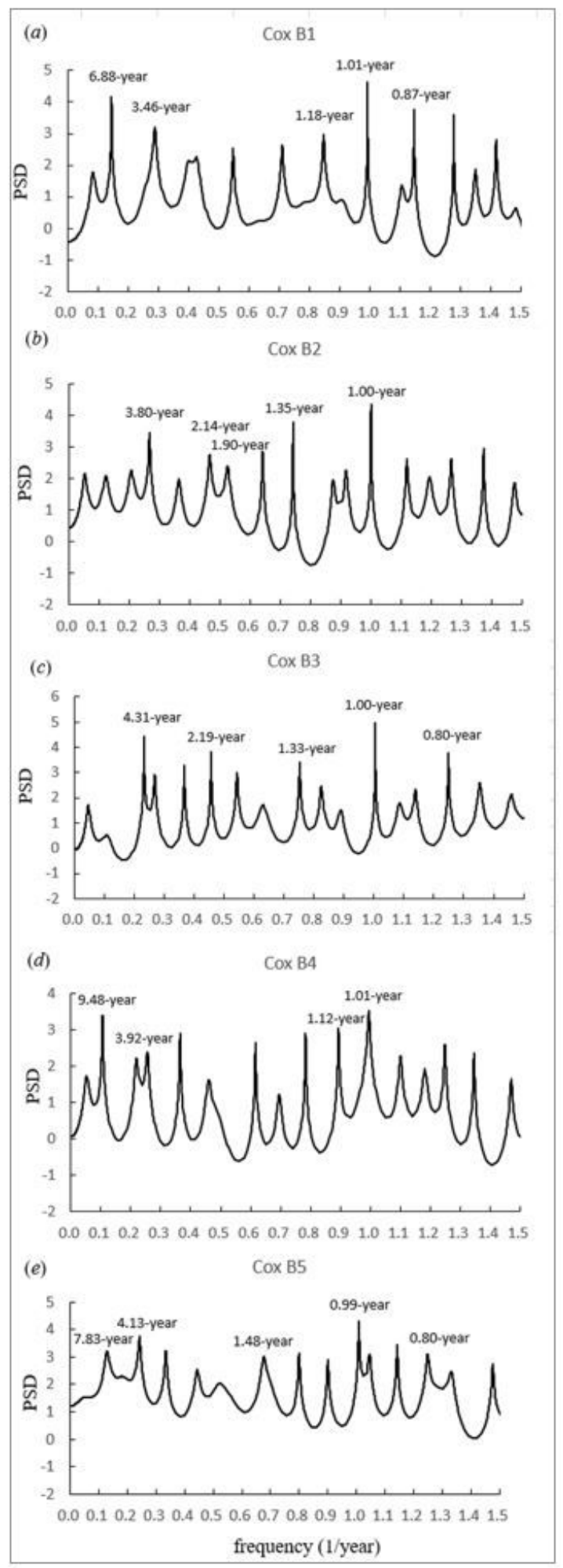

Figure 2

Power spectral density plots of the original data for (a) CVB1, (b) CVB2, (c) CVB3, (d) CVB4, and (e) CVB5.

\section{Supplementary Files}

This is a list of supplementary files associated with this preprint. Click to download. 
- Additionalfile1.pdf

- Additionalfile2.pdf

Page 10/10 